\title{
Modeling Reverse-Phi Motion-Selective Neurons in Cortex: Double Synaptic-Veto Mechanism
}

\author{
Chun-Hui Mo \\ mo@klab.caltech.edu \\ Division of Biology, and Division of Chemistry, California Institute of Technology, \\ Pasadena, CA 91125, U.S.A.

\section{Christof Koch} \\ koch@klab.caltech.edu \\ Division of Biology, Division of Engineering and Applied Science, California Institute \\ of Technology, Pasadena, CA 91125, U.S.A.
}

Reverse-phi motion is the illusory reversal of perceived direction of movement when the stimulus contrast is reversed in successive frames. Livingstone, Tsao, and Conway (2000) showed that direction-selective cells in striate cortex of the alert macaque monkey showed reversed excitatory and inhibitory regions when two different contrast bars were flashed sequentially during a two-bar interaction analysis. While correlation or motion energy models predict the reverse-phi response, it is unclear how neurons can accomplish this. We carried out detailed biophysical simulations of a direction-selective cell model implementing a synaptic shunting scheme. Our results suggest that a simple synaptic-veto mechanism with strong direction selectivity for normal motion cannot account for the observed reverse-phi motion effect. Given the nature of reverse-phi motion, a direct interaction between the $\mathrm{ON}$ and $\mathrm{OFF}$ pathway, missing in the original shunting-inhibition model, it is essential to account for the reversal of response. We here propose a double synaptic-veto mechanism in which $\mathrm{ON}$ excitatory synapses are gated by both delayed $\mathrm{ON}$ inhibition at their null side and delayed OFF inhibition at their preferred side. The converse applies to OFF excitatory synapses. Mapping this scheme onto the dendrites of a direction-selective neuron permits the model to respond best to normal motion in its preferred direction and to reverse-phi motion in its null direction. Two-bar interaction maps showed reversed excitation and inhibition regions when two different contrast bars are presented.

\section{Introduction}

Reverse-phi motion was first demonstrated by Anstis (1970; Anstis \& Rogers, 1975). Subjects perceived the reverse direction of motion when the contrast of a moving object reverses in the second frame of a two-frame 
shift experiment. A repetitive four-stroke cycle of reverse-phi motion gives a strong illusion of unidirectional apparent motion (Anstis \& Rogers, 1986). The reverse-phi illusion involves the short-range motion process pathway (Chubb \& Sperling, 1989). Random dot cinematograms (RDC) studies suggest that $D_{\max }$, the maximum distance dots can move from one frame to the other while still preserving the sense of motion, for reverse-phi motion is comparable to $\mathrm{D}_{\max }$ for normal motion, compatible with the notion that the same short-range direction-selective mechanism is most likely responsible for both normal and reverse-phi motion (Sato, 1989). Reverse-phi-like effects have also been reported during electrophysiological experiments from direction-selective complex cells in cat striate cortex (Emerson, Citron, Vaughn, \& Klein, 1987), the H1 cell in the fly's lobula plate (Egelhaff \& Borst, 1992), and the optical tract of the wallaby (Ibbotson \& Clifford, 2001). Recent recordings from direction-selective cells in the alert monkey show that cells in cortical areas V1 and MT reverse facilitation and suppression regions in the two-bar interaction map when two different contrast bars are presented (Livingstone, Tsao, \& Conway, 2000; Conway \& Livingstone, 2001). This implies that these cells respond to reverse-phi motion in the reversed direction.

Space-time plots of reverse-phi motion show energy in the reverse direction (see Figure 1A). Although the bar movement direction is to the right, the left motion energy unit aligns better with the stimuli and extracts more motion energy than the right motion energy unit. Therefore, both the motion energy model (Adelson \& Bergen, 1985) and the equivalent Reichardt model (Reichardt, 1961; Santen \& Sperling, 1985) can account for the reverse-phi motion. At the core of the Reichardt detector is a correlation step that involves multiplication between inputs. Mathematically, if the sign of one of the inputs being multiplied is reversed, as in the case of reverse-phi motion, the sign of the final product is also reversed. However, there is no experimental evidence that either a single neuron or a neural network can perform a clean, four-quadrant multiplication operation. Even for a single identifiable cell that performs multiplication, such as the LGMD neuron in the locust's visual system (Hatsopoulos, Gabbiani, \& Laurent, 1995; Gabbiani, Mo, \& Laurent, 2001), it is unlikely that the sign of the cell's output could be reversed for a reversed signed input, given multiple half-wave rectification mechanisms and narrow operating ranges for most biophysical processes involved. It is therefore of interest to study a direction-selective mechanism that can be implemented by neurons and can account for both normal and reverse-phi motion.

The first computational step in visual processing is half-wave rectification and separation into $\mathrm{ON}$ and OFF channels. It is unknown whether direction selectivity is generated between nonlinear interactions of these half-wave rectified signals or between simple cells that carry the reconstructed full wave signal. DL-2-amino-4-phosphonobutyric acid (APB) reversibly blocks the ON response in the mammalian retina (Schiller, 1982), but the detection of motion direction is largely unaffected in rabbit, cat, and monkey in electro- 
A

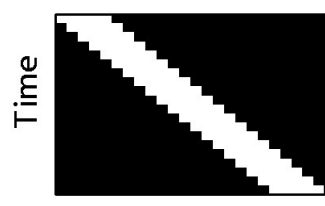

Space

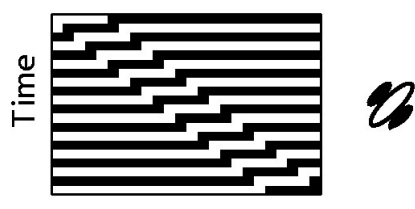

S pace

B

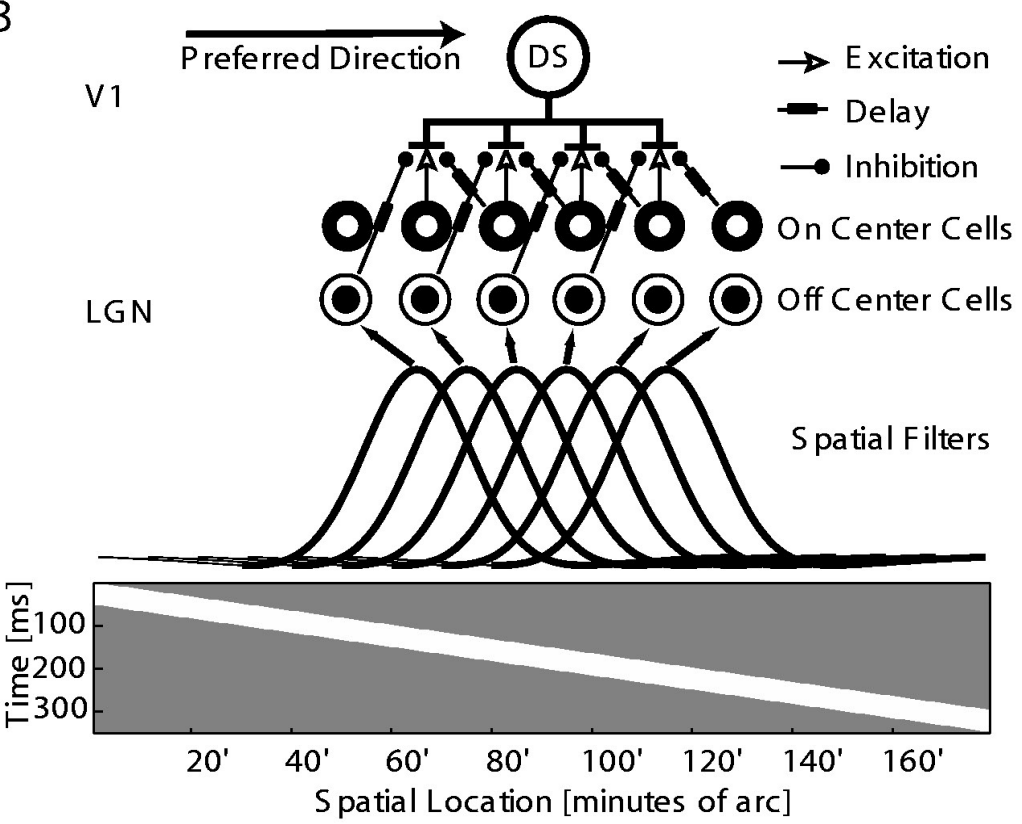

Figure 1: Space-time plot of normal and reverse-phi motion and connectivity diagram of the model that accounts for direction and reverse-phi selectivity. (A) Space-time plot of a one-dimension white bar moving from left to right in normal motion (left panel) and reverse-phi motion (right panel). A rightmotion energy unit aligns well with the normal motion plot, but triggers a much reduced response for the reverse-phi motion. Instead, this strongly stimulates a left motion energy unit. (Adapted from Fig. 16 in Adelson \& Bergen, 1985.) (B) Connectivity diagram of normal and reverse-phi motion direction-selective model. Input to LGN neurons comes from a one-dimensional array of 179 pixels. The intensities from those pixels were summed through difference of gaussian spatial filters on to LGN cells. There are one ON and one OFF center geniculate cell at one of six spatially offset locations. Each of the middle four ON center LGN cells provided excitatory input to one branch of the model cell dendrites, while the ON center LGN cell immediately to the right and the OFF center LGN cell immediately to the left provide delayed on-the-path inhibition. The converse connection scheme for the OFF center LGN cells' excitatory inputs is not shown. 
physiological experiments (Knapp \& Mistler, 1983; Horton \& Sherk, 1983; Schiller, Sandel, \& Maunsell, 1986). Similarly, existing direction-selective models treat ON and OFF inputs separately (Koch \& Poggio, 1985; Suarez, Koch, \& Douglas, 1995; Rao \& Sejnowski, 2000). However, the nature of reverse-phi motion stimuli suggests an interaction between ON and OFF channels. Different rectification schemes affect the ON-OFF interaction differently, and therefore carefully constructed reverse-phi stimuli were used in experiments to separate the first-order, second-order, and third-order motion (Lu \& Sperling, 1999; Mather \& Murdoch, 1999). The requirement for ON-OFF interactions constrains cellular models of direction selectivity.

V1 direction-selective cells show slanted excitatory regions in their receptive field map (Livingstone, 1998). The asymmetry in the summation of excitatory inputs at dendrites alone was not sufficient to account for the directional response based on a modeling study by Anderson, Binzegger, Kahana, Martin, and Segev (1999). Therefore, asymmetrical delayed inhibition is likely to be the mechanism that underlies direction selectivity. Such a mechanism based on shunting inhibition (i.e., an increase in a chlorine-based $\mathrm{GABA}_{\mathrm{A}}$ conductance that reverses close to the cell's resting position) was proposed for the cortex by Koch and Poggio (1985). Recent experiments in the retina provide evidence in favor of at least some nonlinear interactions between excitatory and shunting inhibitory inputs that take place within the dendrites of direction-selective ganglion cells (Taylor, He, Levick, \& Vaney, 2000; for a dissenting view, see Borg-Graham, 2001). Large conductance changes that reverse around the cell's resting potential have been observed in V1 during visual stimuli (Anderson, Carandini, \& Ferster, 2000; Borg-Graham, Monier, \& Frégnac, 1998). Here we show how a double synaptic-veto mechanism, derived from the traditional asymmetrical delayed shunting inhibition model, can account for both normal and reverse-phi motion direction selectivity.

\section{Methods}

We followed a two-step compartmental simulation strategy using the program NEURON (Hines \& Carnevale, 1997). We first investigated the performance of an idealized neuron (see Figure $4 \mathrm{~B}$ ) before we implemented our synaptic assembly onto a reconstructed V1 cell (see Figure 7A). The idealized cell morphology is shown in Figure 4B. Eight dendrites (width, $0.5 \mu \mathrm{m}$; length, $100 \mu \mathrm{m}$ ) were directly connected to the soma (width, $16 \mu \mathrm{m}$; length, $16 \mu \mathrm{m})$. Each dendrite had 20 compartments, for a total of 180 compartments. The dendrites were passive, while the cell body contained a number of voltage-dependent currents that give rise to fast Hodgkin-Huxley-like action potentials. The biophysical parameters were as follows: $R_{a}=250$ $\Omega \bullet \mathrm{cm}^{2}, \mathrm{C}_{\mathrm{m}}=0.5 \mu \mathrm{F} / \mathrm{cm}^{2}, \mathrm{E}_{\text {leak }}=-60 \mathrm{mV}, R_{\mathrm{m}}=10 \mathrm{k} \Omega \bullet \mathrm{cm}, g_{\mathrm{Na}}=0.024$ $\mathrm{S} / \mathrm{cm}^{2}, \mathrm{~g}_{\mathrm{K}}=0.020 \mathrm{~S} / \mathrm{cm}^{2}, \mathrm{E}_{\mathrm{NMDA}}=0 \mathrm{mV}, \mathrm{g}_{\mathrm{NMDA}}=2.5 \mathrm{nS}, \tau_{\mathrm{NMDA}}$ on $=0.1$ $\mathrm{ms}, \tau_{\mathrm{NMDA} \text { off }}=80 \mathrm{~ms}, \mathrm{E}_{\mathrm{AMPA}}=0 \mathrm{mV}, \mathrm{g}_{\mathrm{AMPA}}=2.5 \mathrm{nS}, \tau_{\mathrm{AMPA}}$ on $=0.1$ 
$\mathrm{ms}, \tau_{\mathrm{AMPA} \text { off }}=2 \mathrm{~ms}, \mathrm{E}_{\mathrm{GABA}}=-60 \mathrm{mV}, \mathrm{g}_{\mathrm{GABA}}=6.0 \mathrm{nS}, \tau_{\mathrm{GABA}}$ on $=1 \mathrm{~ms}$, $\tau_{\mathrm{GABA} \text { off }}=80 \mathrm{~ms}$. Synaptic input was modeled using the point process in NEURON (adopted from Archie \& Mel, 2000).

We adapted a layer 4 stellate cell model from Mainen and Sejnowski (1996; see Figure 7A). The model cell contained sodium channels at the soma and dendrites, as well as fast potassium channels at the soma and the axon. Both calcium- and voltage-dependent slow potassium channels and high-threshold calcium channels were present at the soma and dendrites. All passive and active parameters were the same as those used in their original paper. All synapse parameters were the same as described above except $\mathrm{E}_{\mathrm{GABA}}=-70 \mathrm{mV}, \mathrm{g}_{\mathrm{GABA}}=6.9 \mathrm{nS}$.

The input connection scheme to both models is shown in Figure 1B. The spatial resolution of the stimulus was 1 minute of arc and the temporal resolution $0.1 \mathrm{~ms}$. The lateral geniculate nucleus (LGN) layer was modeled as a transfer function. Stimuli projected onto the LGN layer consisted of two $6 \times 1$ arrays of spatial filters modeling ON center and OFF center cells that covered 3 degrees of visual space. The spatial kernel was a difference of gaussian (DOG) function (adapted from Wörgötter \& Koch, 1991). The gaussian kernel was $\mathrm{G}(\mathrm{x})=\left(\mathrm{K} / 2 \pi \sigma^{2}\right) \exp \left(-\mathrm{x}^{2} / 2 \sigma^{2}\right), \sigma_{\text {center }}=10.6$ minute, $\sigma_{\text {surround }}=31.8$ minute, and $\mathrm{K}_{\text {center }} / \mathrm{K}_{\text {surround }}=17 / 16$. Stimuli were first passed through these spatial filters and then through low-pass temporal filters, with a delay between the center and the surround component $\left(\tau_{\text {center }}=10 \mathrm{~ms}, \tau_{\text {surround }}=20 \mathrm{~ms}\right.$ delay surround $\left.=3 \mathrm{~ms}\right)$. The resulting values were scaled to give a time-dependent, stimulus-driven LGN instantaneous firing rate with a maximum value of $200 \mathrm{~Hz}$. A background firing rate of $5 \mathrm{~Hz}$ was added, and all negative values were set to 0 (half-wave rectification). OFF center cells were modeled as the reverse of their ON center counterpart.

Each of the middle four ON center LGN cells provided the excitatory input to one branch of the model cell dendrites, while the ON center LGN cell immediately to the right (preferred side) and the OFF center LGN cell immediately to the left (null side) provided delayed inhibition. Each of the middle four OFF center LGN cells provided excitatory input to one branch of the model cell dendrites, while the OFF center LGN cell immediately to the right and the ON center LGN cell immediately to the left provided delayed inhibition. The delay was $12 \mathrm{~ms}$. There were 8 excitatory synapses and 16 inhibitory synapses in the model. Excitatory synapses were mapped to the dendrite compartment $60 \mu \mathrm{m}$ away from the soma; the same type of inhibitory input was located $50 \mu \mathrm{m}$ and a different type of inhibition $40 \mu \mathrm{m}$ away from the cell body. Given that shunting inhibition was on the direct path between excitation and the soma, it could effectively and specifically veto the excitatory input to that branch while only minimally affecting the excitatory input from neighboring branches (Koch, Poggio, \& Torre, 1982).

For the layer 4 stellate cell, eight triplets of excitatory-inhibitoryinhibitory synapses were mapped to eight separate terminal branches. This 
arrangement was replicated four times and mapped onto 32 terminal branches. The total synaptic count was 32 excitatory and 64 inhibitory synapses.

\section{Results}

We started by testing how well the reverse-phi effect was explained by the original asymmetric-delayed inhibition model of Barlow and Levick (1965), as implemented with shunting inhibition (Torre \& Poggio, 1978; Poggio \& Torre, 1978; Koch et al., 1982). We then carried out compartmental simulation in NEURON using an idealized dendritic geometry to prove our concept and compare the model against experimental data. Finally, we mapped our synaptic connection scheme to a more realistic cortical cell morphology and demonstrated how it could account for both normal and reverse-phi direction selectivity.

3.1 Asymmetric-Delayed Shunting Inhibition Model. The traditional Barlow and Levick (1965) inhibitory-based scheme is shown in Figure 2A. Inhibition was assumed to be of the shunting type (that is, with an inhibitory reversal potential around the local resting potential) so that synaptic interactions are restricted to local branches. We plotted the time course of excitatory and inhibitory inputs to the model from two adjacent cells in the LGN input layer (see Figure 2B). When a white bar moved in a normal fashion in the preferred direction, there was a temporal shift between excitatory and inhibitory inputs (see Figure 2B.a), while in the null direction, excitation and inhibition overlapped and therefore cancelled each other (see Figure 2B.c). If the same bar moved in the reverse-phi fashion, there was little difference in the temporal alignment of excitatory and inhibitory inputs between preferred and null direction movement (see Figures 2B.e-h). For normal motion

Figure 2: Facing page. Normal shunting inhibition cannot account for reversephi motion. (A) Asymmetrical delayed inhibition scheme resulted in direction selectivity. $\mathrm{ON}$ excitation was gated by a delayed $\mathrm{ON}$ inhibition at its preferred side and the converse for OFF excitation (symbols as in Figure 1). (B) Excitatory and inhibitory inputs to model cell when a bright thin bar moves across its receptive field at 10 degrees per second in preferred normal $(\mathrm{PN})$ motion direction $(\mathrm{a}, \mathrm{b})$; in null normal $(\mathrm{NN})$ motion direction $(\mathrm{c}, \mathrm{d})$, preferred reverse-phi (PR) motion direction $(\mathrm{e}, \mathrm{f})$, and null reverse-phi $(\mathrm{NR})$ motion direction $(\mathrm{g}, \mathrm{h})$. Solid lines: Excitatory inputs from ON center cells $(a, c, e, g)$ and OFF center cells $(b, d, f, h)$. Dashed lines: Delayed inhibitory inputs (20 ms delay) were plotted in negative. Inputs were calculated as the stimuli passed through the spatial-temporal filters mentioned in section 2. (C) The direction index DI for the eight-armed dendritic model cell for different inhibitory delay times and contrast reversal rates. Over a wide range of inhibitory input delay times, the model cell was direction selective to normal motion but only weakly to reverse-phi motion. 


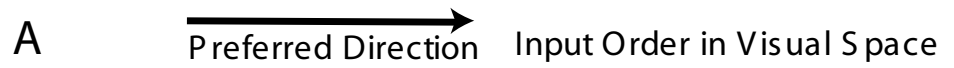
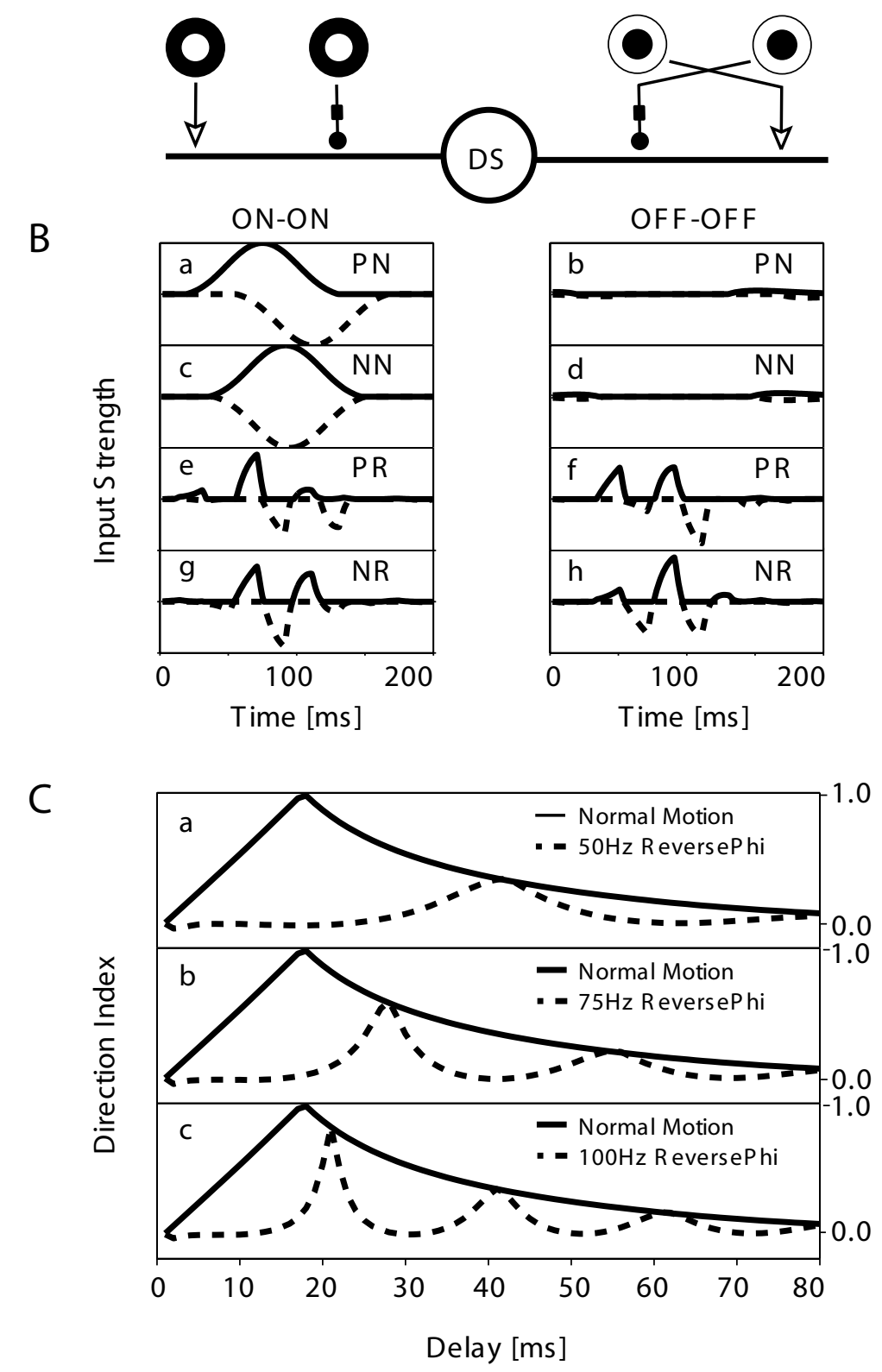
only, the ON branch received significant input, while for reverse-phi motion, the input was spread between $\mathrm{ON}$ and OFF channels. The combined areas under the excitation or inhibition curves for both branches were much less for reverse-phi motion than normal motion. Because of the low-pass filtering and the rectification inherent in the LGN layer, both the excitatory and inhibitory inputs to the model cell during reverse-phi motion were reduced.

We compared the direction index (DI) of the model cell using different stimulus reversal rates and the delay factor for the inhibition (see Figure 2C). DI was calculated as the response in the preferred direction minus the null direction response, divided by the sum of the preferred and the null direction. The value of preferred direction was calculated as the sum of excitation minus inhibition, with all negative values set to zero. Thus, DI ranges from 0 (for a nondiscriminatory system) to 1 (for a system that does not respond at all to null direction motion). The model was direction selective to normal motion for a wide range of delay values but only weakly direction selective to reverse-phi motion. DI for reverse-phi motion increased when the stimulus reversal rate increased, but the direction preference was the same as that of normal motion, contrary to the experimental data. Therefore, it appears that a traditional inhibition scheme cannot easily account for reverse-phi motion.

Given the nature of reverse-phi motion, a direct nonlinear interaction between $\mathrm{ON}$ and OFF branches, missing in traditional schemes, is needed to account for reverse-phi motion-direction selectivity. We here propose a "reversed" shunting inhibition scheme (see Figure 3A), in which an ON excitation is gated by a delayed OFF inhibition at its null side and an OFF excitation is gated by a delayed ON inhibition at its null side (see Poggio, 1982; Koch \& Poggio, 1987, for other synaptic logic models involving ONOFF interaction). Not surprisingly, this model responds equally strongly to both directions for normal motion, as inhibition is activated only by a bar of the opposite contrast from that of excitation. However, there is a difference in the temporal alignment of excitatory and inhibitory inputs between the

Figure 3: Facing page. Reversed shunting inhibition scheme was direction selective to reverse-phi motion. (A) Reversed asymmetrical delayed-inhibition scheme. ON excitation was gated by a delayed OFF inhibition at its preferred side and the converse for OFF excitation. (B) Excitatory and inhibitory inputs to the model cell when a bright thin bar moves across its receptive field at 10 degrees per second in the preferred normal $(\mathrm{PN})$ motion direction $(\mathrm{a}, \mathrm{b})$; the null normal (NN) motion direction (c,d), the preferred reverse-phi (PR) motion direction $(\mathrm{e}, \mathrm{f})$, and the null reverse-phi $(\mathrm{NR})$ motion direction $(\mathrm{g}, \mathrm{h})$. Solid lines: Excitatory inputs from ON center cells $(a, c, e, g)$ and OFF center cells $(b, d, f, h)$. Dashed lines (delayed inhibitory inputs-20 ms delay) are plotted in a reverse negative. (C) The DI calculated for reverse-phi motion. The model is not direction selective to normal motion, but when inhibitory input delay is optimal, the model was direction selective to reverse-phi motion. 


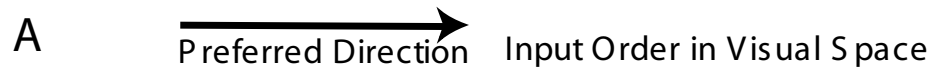

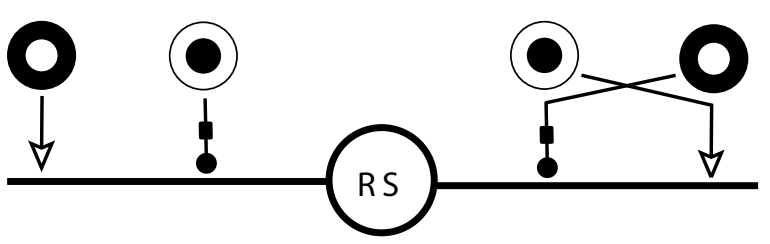

B
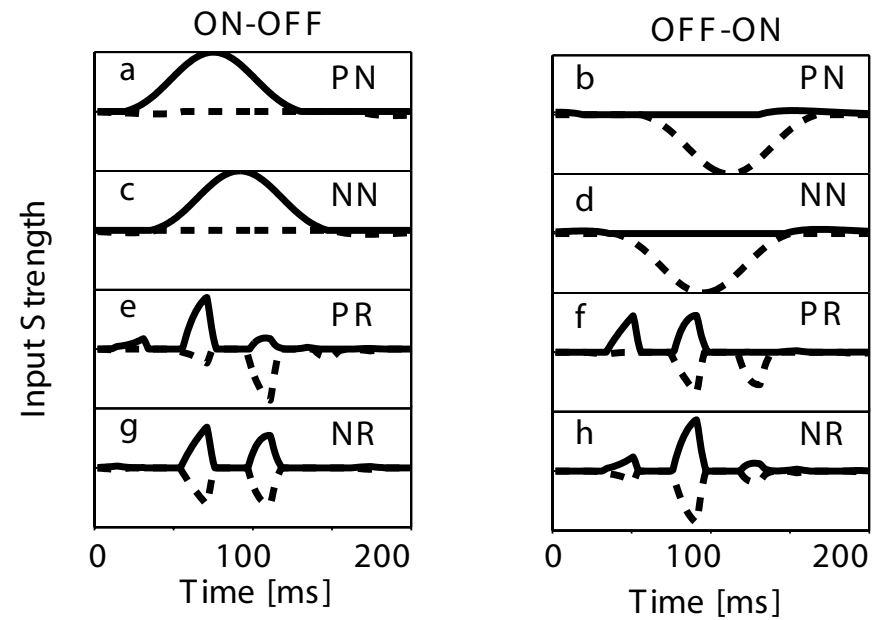

C

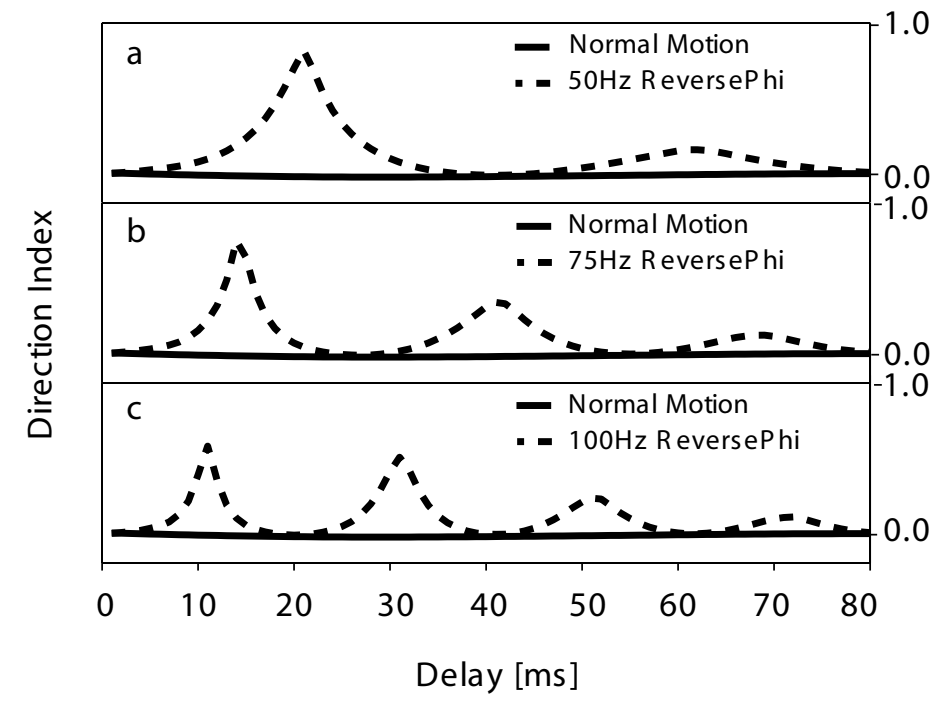


preferred and null direction reverse-phi movement. When the inhibitory delay matches the stimuli reversal rate, DI is close to 1 . DI decreases when the stimulus reversal rate increases. We conclude that the ON-OFF and OFF$\mathrm{ON}$ vetoing scheme can discriminate the direction of reverse-phi motion. Given that the experimental data demonstrated that reverse-phi motion in the cell's null direction elicited more vigorous responses than reverse-phi motion in the preferred direction $(\mathrm{DI}<0)$, the delayed inhibitory input needs to reside at the excitatory input's preferred side instead of its null side.

3.2 Double Synaptic-Veto Mechanism. A traditional shunting inhibition scheme can account for normal motion-direction selectivity, while a "reversed" shunting inhibition scheme can account for reverse-phi motiondirection selectivity. In order to account for both, we need to combine both synaptic schemes. One way to achieve this is to construct a model cell with four dendritic branches. Two of them implement a traditional shunting inhibition scheme and the other two a "reversed" shunting inhibition scheme. Such a connection scheme will be selective to both types of motion; however, the "traditional" branches offer a nongated path for reverse-phi excitatory inputs, while the "reversed" branches offer a nongated path for normal motion excitation. Such nongated excitatory inputs result in a high background level of depolarization at the soma and therefore low DI values.

We propose a double synaptic-veto mechanism that combines the two synaptic schemes in a more sophisticated way at the microcircuitry level (see Figure 4A). In the new connection scheme, an ON excitatory synapse is gated by both a delayed ON inhibition at its null side (see Figure 1B, right side of the cell) and a delayed OFF inhibition at its preferred side (see Figure 1B, left side of the cell), and an OFF excitatory synapse is gated by both a delayed OFF inhibition at its null side and a delayed ON inhibition at its preferred side. Same-type inhibition at the null side vetoes nulldirection normal motion, while different-type inhibition at the preferred side vetoes preferred-direction reverse-phi motion. We mapped this triplet of synapses-one excitatory and two shunting inhibitory ones-onto the

Figure 4: Facing page. A double synaptic-veto mechanism can account for both normal and reverse-phi motion direction selectivity. (A) Connection scheme of the double synaptic veto mechanism. ON excitation is gated by a delayed $\mathrm{ON}$ inhibition at its preferred side and a delayed OFF inhibition at its null side; the converse is true for OFF excitation. (B) Mapping the double synaptic-veto scheme onto the eight-armed cable model with spiking at the cell body. Each excitation-inhibition-inhibition triplet is mapped onto its own dendritic branch with the excitation at the far side of cell body. (C) Model cell's response to a bright bar moving at 10 degrees per second across its receptive field. The model cell responds best when a normal motion stimulus moves in its preferred direction and a reverse-phi motion stimulus moves in its null direction. The stimuli reverse rate is $75 \mathrm{~Hz}$. 
A Input Order in Visual S pace B
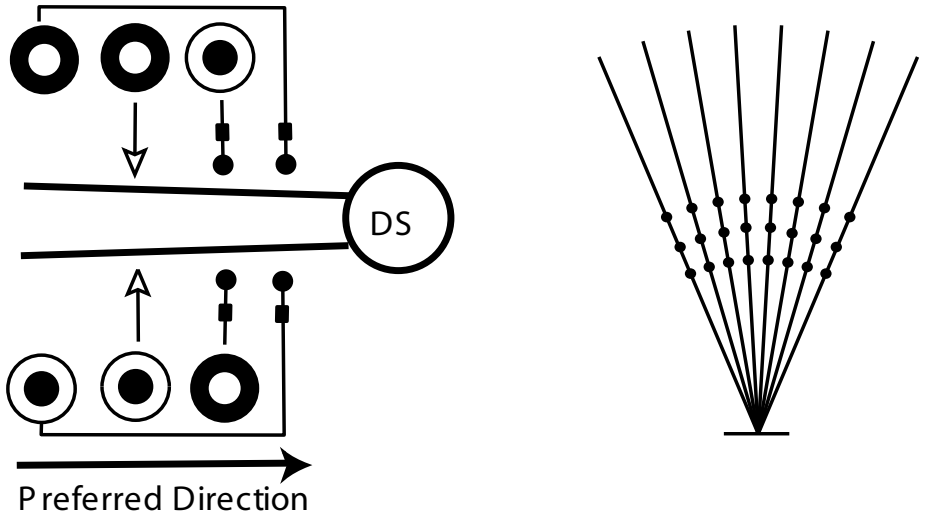

C
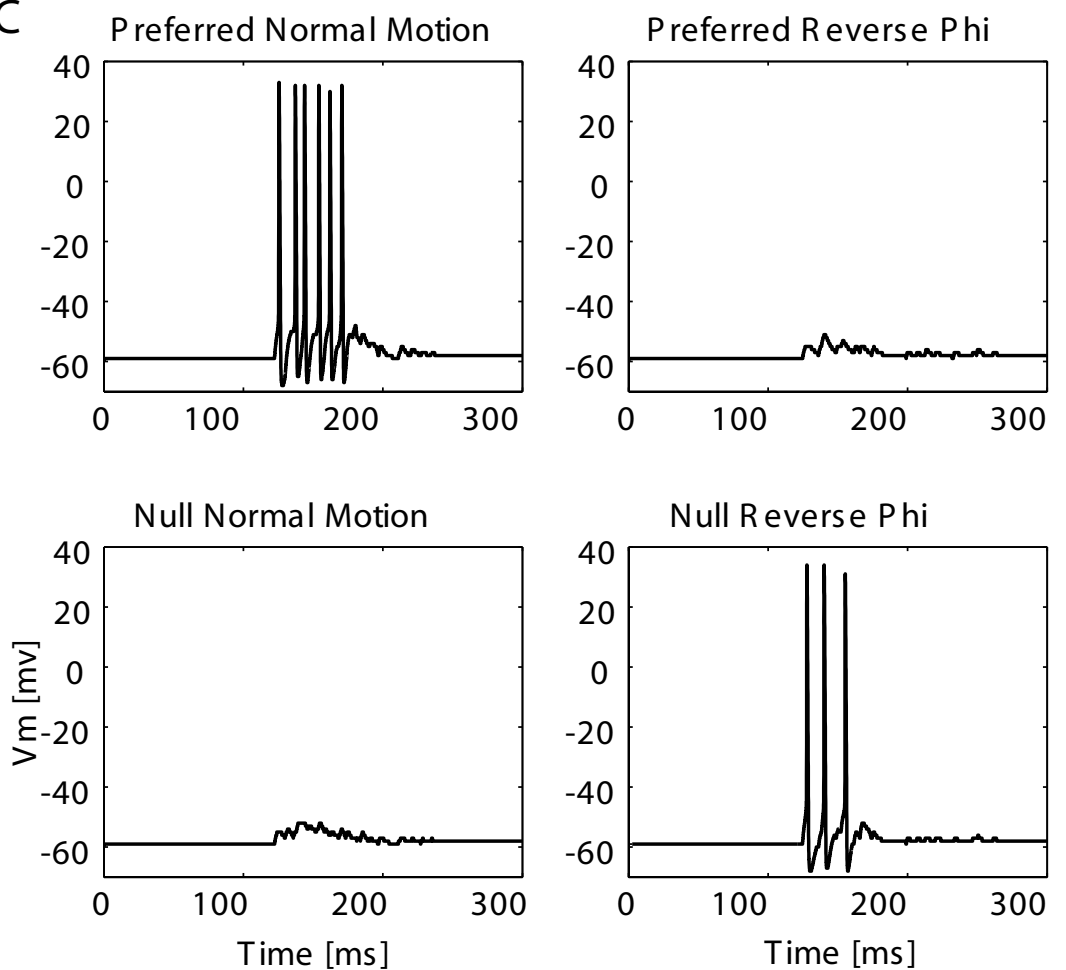
abstract cell model with a soma and eight dendritic branches (see Figure 4B), creating a simulacrum with four direction-selective subunits, each of which implements the synaptic connection of Figure 4A (the detailed connection scheme is explained in section 2).

The model responded best to normal motion in its preferred direction $(D I=1)$ and reverse-phi motion ( $D I=-1)$ in its null direction (see Figure $4 C$ ). Note that the amplitude of the response to normal motion (6 spikes) was twice as large as the amplitude to reverse-phi motion ( 3 spikes), reflecting the fact that both the excitatory and inhibitory inputs for normal motion stimuli were stronger. In a broad range of model parameters, $\mathrm{Cm}=0.5$ to $1 \mu \mathrm{F} / \mathrm{cm}^{2}$, $\mathrm{E}_{\text {leak }}=-70$ to $-60 \mathrm{mV}$, Delay inhibitory input $=8$ to $15 \mathrm{~ms}, \mathrm{~g}_{\mathrm{AMPA}}+\mathrm{g}_{\mathrm{NMDA}}=1$ to $10 \mathrm{nS}, \mathrm{g}_{\mathrm{AMPA}} / \mathrm{g}_{\mathrm{NMDA}}=0.1$ to 10 , and $g_{\mathrm{GABA}} /\left(\mathrm{g}_{\mathrm{NMDA}}+\mathrm{g}_{\mathrm{NMDA}}\right)=1$ to 10 , the simulation showed direction selectivity for both types of motion, but only specific parameter sets resulted in high DI. When DI was small, null-direction normal motion or preferred-direction reverse-phi motion also elicited spikes, but the timing of the first spike was late compared to the case of the preferred-direction movement (data not shown). Even if g NMDA was set to zero, the cell responded in a differential way to null- and preferreddirection motion. Since sodium and potassium channels are placed only at the soma, the voltage-dependent dendritic current was not required for the model's direction selectivity. However, NMDA currents increased somatic voltage during preferred-direction movement and thus increased DI. The inhibitory synapses were always located between the excitatory input and the spike-triggering zone at the cell body, thereby fulfilling the "on-thepath" condition (Koch et al., 1982). The inhibitory conductance change in most cases needs to be only a little bit larger than the excitatory conductance change to achieve a "veto" effect.

To test whether shunting inhibition was required for the direction selectivity we observed, we decreased the GABA channel reversal potential from $-60 \mathrm{mV}$ to $-90 \mathrm{mV}$ in $5 \mathrm{mV}$ steps. At the same time, we decreased the amplitude of $g_{\text {GABA }}$ accordingly so that the model always responded to a preferred-direction normal-motion stimuli with six spikes and nulldirection reverse-phi stimuli with three spikes. DI for both types of motions decreased when the GABA channel reversal potential was decreased. At $-90 \mathrm{mV}$, the model responded with five spikes to null-direction normalmotion stimuli and with three spikes to preferred-direction reverse-phi motion stimuli. Thus, the direction selectivity was lost.

3.3 Receptive Field and Two-Bar Interaction Maps. The direction-selective cell shows a slant in the space-time plot (see Figure 5A). A recent study of direction-selective cells in awake monkey V1 shows an excitatory region on the cell's preferred side and an inhibitory region on the cell's null side, consistent with our connection scheme (Livingstone, 1998). To compare the experimental data with our model, we incorporated a synaptic noise source (AMPA conductance only) at the soma to achieve a reasonably 

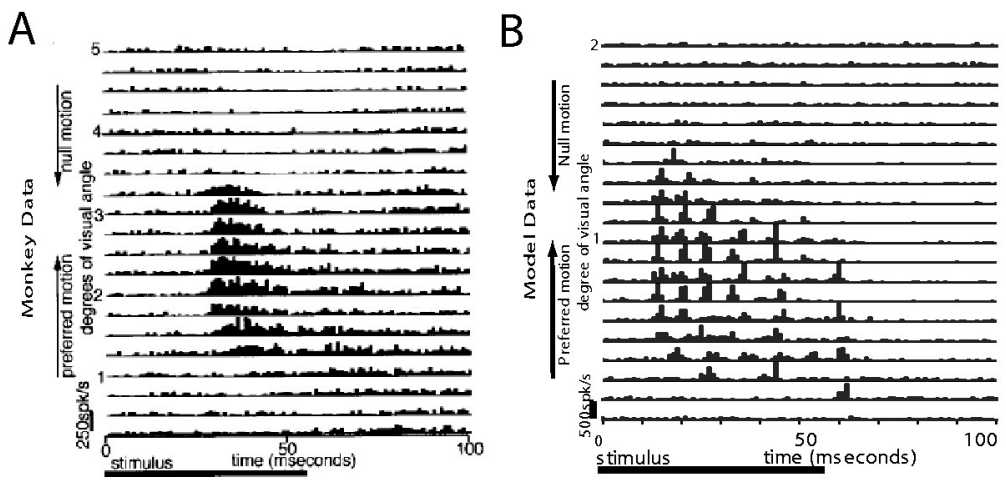

Figure 5: Comparison of space-time response mapping of monkey V1 cell and model cell. (A) Poststimulus time histogram (PSTHs) obtained from a layer 5/6 complex cell in primary visual cortex of the alert macaque monkey in response to flashed bars, presented in random order at a series of positions across the cell's receptive field. (From Livingston, 1998, Fig. 3A.) This cell's preferred direction was from the bottom toward the top. Flash bar duration $=56 \mathrm{~ms}$; interstimulus delay $=100 \mathrm{~ms} ; 75$ stimulus presentations. (B) PSTHs obtained from the model neuron to flashed bars at 20 spatial locations across its receptive field. The model's preferred direction is from the bottom toward the top. It shows a decrease in the response onset time and an increase in the response transiency, as does the V1 complex cell.

high background firing rate and then presented flashing bar stimuli at 20 different spatial locations across the receptive field (see Figure 5B). There was good agreement between the experimental data and the response of our eight-arm model. Both space-time plots showed a progressive shortening of the response onset time and a more transient response going from the cell's preferred side to its null side. In our model, the shortening of response onset time and the increase in response transiency were due to asymmetrical delayed inhibition and the basic property of integrate-and-firing neurons of LGN layer. The response onset time was determined mainly by the excitatory input since the inhibitory input was relatively small and delayed. Going from the preferred to the null side, the bar moves from the edge to the center of the receptive field of the first LGN cell that provided excitatory input, increasing the excitatory input amplitude and decreasing the time needed to charge the membrane to fire the first spike. The response transiency is determined primarily by how quickly inhibition can overcome excitation and shut off the response. Moving toward the null side, excitatory input strength decreases, while inhibitory input strength increases, as does the response transiency. The slant of the excitatory and inhibitory regions in the space-time plot is related to the cell's velocity tuning (Livingstone, 1998; 
McLean \& Palmer, 1989; Reid, Soodak, \& Shapley, 1991). Since we did not include any synaptic delay between the retina and V1, the model responds much earlier to the visual stimulus than actual striate cortex neurons do. The periodic firing is caused by the deterministic synaptic input. Random noise channels at the soma are responsible for the background firing and jitter of spikes around the peak firing time. Rao and Sejnowski (2000) showed a similar space-time plot for their direction-selective model. In their network model, the decrease of response onset time was due to the increase of the excitatory synaptic input strength from the cell at the preferred side toward the model cell itself. This effect is expected for any direction-selective model based on asymmetrical inhibition.

The experimental data also show reversed excitatory and inhibitory regions in the two-bar interaction map of direction-selective cells in V1 and MT when opposite-contrast bars, instead of same-contrast bars were presented (MT: see Figure 6A, from Livingstone, 2001, Fig. 1; V1: Livingstone,

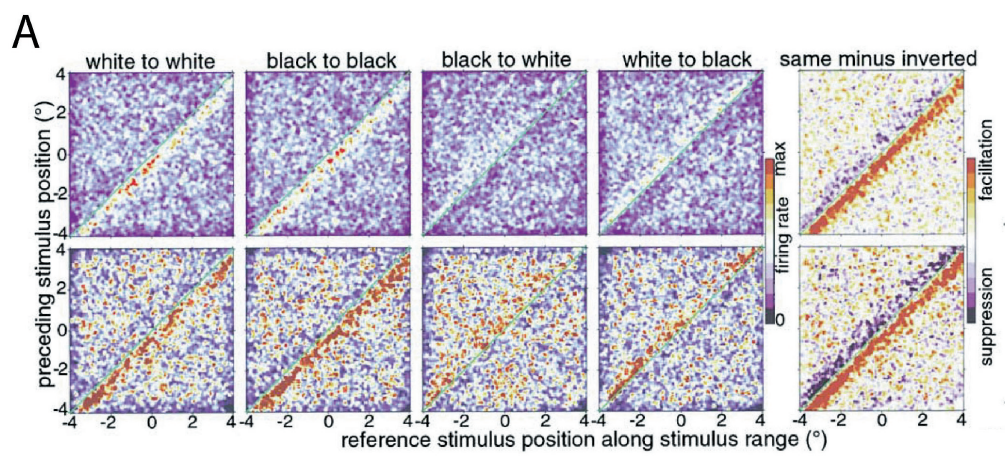

B
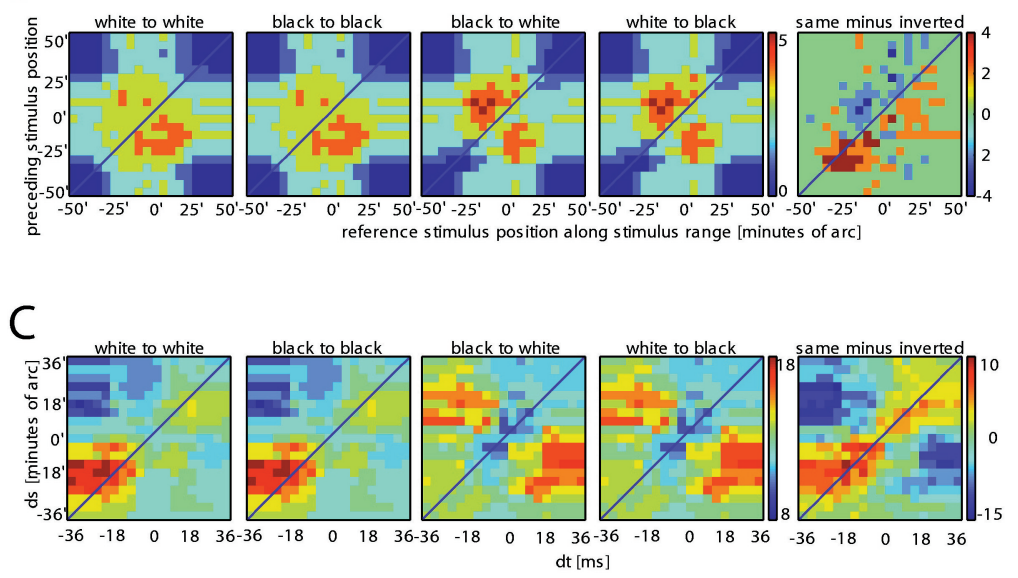
personal communication, April 2002). To compare with the experimental data, we tested our model's response to two sequentially presented bars (see Figure 6B). The $x$-axis corresponds to the position of the first flashed bar and the $y$-axis to the position of the second flashed bar. The diagonal line corresponds to both bars being presented at the same spatial location. A nondirection-selective cell should have excitation regions both above and below the diagonal line, while a direction-selective cell should have asymmetric excitation regions with respect to the diagonal line. When the two bars are of the same contrast, the excitatory region of our model cell's response map is mostly above the diagonal, where the second presenting

Figure 6: Facing page. Two-bar interaction maps. (A) Interaction map for two MT cells in the alert monkey (Fig. 1 in Livingstone et al., 2001). (B) Same interaction map for the eight-armed model neuron. Pairs of bars (8 minutes of width) were flashed sequentially for $13 \mathrm{~ms}$ each at different spatial locations. Spikes were counted for $100 \mathrm{~ms}$ from the start of the first bar flash. The $x$-axis is the first flash bar's position, and the $y$-axis is the second bar's position. The diagonal line is where two bars presented at the same spatial location. From left to right, Panel 1: Both bars were white. Areas above the diagonal line, where the second bar position was more positive than the first bar position, were more active than the area below the diagonal line. Panel 2: Both bars were black. Panel 3: The first bar was black and the second bar was white. Areas below the diagonal line were more active than the area above the diagonal line. Panel 4: The first bar was white, and the second was black. Panel 5: The same contrast conditions minus the inverted contrast conditions $(1+2-3-4)$. The model neuron preferred two same-contrast bars flashed sequentially in its preferred direction and two inverted-contrast bars flashed sequentially in its null direction. Given the symmetrical input, the white-to-white and black-to-black model interactions are identical, as are the black-to-white and the white-to-black one. (C) Space-time two-bar interaction map of the model cell following the technique pioneered by Emerson et al. (1987). The reference bar was presented at time 0 at four different locations across the receptive field. For each reference bar position, the probing bar was presented at different locations and times relative to the reference bar. Spikes were counted for $100 \mathrm{~ms}$ from the start of the first bar flash. Four such maps were added together to give a position-invariant ds-dt map of two bar interactions. From left to right, Panel 1: Both bars were white. Areas along the diagonal line, where the two-bar presenting sequence matched the preferred direction and speed, were more active than areas orthogonal to the diagonal line, where the two-bar presenting sequence matched the null direction. Panel 2: Both bars were black. Panel 3: The reference bar was black, and the probing bar was white. Panel 4: The reference bar was white, and the probing bar was black. Panel 5: Same-contrast conditions minus the inverted-contrast conditions. The model neuron preferred two same-contrast bars flashed sequentially in its preferred direction and two inverted-contrast bars flashed sequentially in its null direction. 
bar's position is located more toward the null side of the first presenting bar. However, when the two bars have opposite signs of contrast, the excitatory region is mostly below the diagonal, whereas the second presenting bar's position is located more toward the preferred side of the first bar, signaling a reverse in the direction preference of the model. There is no difference between the white-white and black-black plots, given the symmetric ON and OFF inputs the model receives (the same is true for the black-white and black-white plots). The difference of the excitatory and inhibitory regions between the same- and inverted-contrast bar presentations is more clearly evident in Figure 6B: excitation is mostly above and inhibition mostly below the diagonal. Note that excitation was stronger at the model cell's preferred side (minus side of spatial scale), while inhibition was stronger at the model cell's null side (plus side of spatial scale). This was again due to the spatial asymmetry of excitation and inhibition. Although the model is direction selective to both presentations, direction selectivity was higher for samecontrast bar presentations than for different-contrast bar presentations. This translates into a weaker response to reverse-phi than to normal motion. The experimental data from MT cells in Figure 6A show the same overall trend but with a much larger receptive field and better overall position invariance across the receptive field. There were a few major differences between the empirically determined MT cell response maps (see Figure 6A) and our V1 model cell response (see Figure 6B). Figure 6A shows a diagonal organization, while Figure 6B shows a more circular organization. Part of this difference can be explained by the receptive-field size difference between MT and V1 cells. This difference can also arise due to differences in the number and density of direction-selective subunits along the preferred direction and the extent of the nonlinear boost of the final output stage. Some V1 direction-selective complex cells do show a circular interaction region, while other V1 complex cells reveal a more diagonal organization (M. Livingstone, personal communication, April 2002). In addition, the diagonal region in the model (see Figure 6B) is above baseline, whereas it appears to be below baseline in the data (see Figure 6A). This elevation is likely due to the reverse-correlation technique used in the experiment and further nonlinear excitation mechanisms that are missing from our model.

Despite these differences, the "same minus inverted" maps (the rightmost panel in Figures 6A and 6B), which demonstrate the nonlinear interactions, do show a substantial amount of similarity. Finally, the cross-shaped backdrop in Figure 6B does not appear in the experimental data. Again, this is due to the difference in receptive-field size between the recorded MT cell and our V1 model cell. If Figure 6A were evaluated within a larger spatial range (for example, within $+/-10$ degrees), the same cross-shaped background would appear (M. Livingstone, personal communication, April 2002).

We also generated space-time two-bar interaction maps (see Figure 6C) to compare with the experimental data published by Emerson et al. (1987) on 
complex cells in cat striate cortex. The $x$-axis corresponds to the time of the flashed probe bar relative to the time of presentation of the flashed reference bar. The $y$-axis corresponds to the position of the flashed probe bar relative to the position of the flashed reference bar (for more details, see Emerson et al., 1987). A direction-selective cell should have obliquely oriented excitatory regions (Figure 2 in Emerson et al., 1987). When the two bars are of the same contrast, the excitatory region of our model cell's response map is mostly found along the diagonal, whereas the second presenting bar's position is located more toward the null side of the first presenting bar. However, when the two bars presented have opposite contrast sign, the excitatory region mostly flanks the diagonal area, whereas the second presenting bar's position is located more toward the preferred side of the first bar, signaling a reverse in the direction preference of the model. The difference of excitatory and inhibitory regions between the same and inverted contrast bar presentations is more clearly evident in the "same minus inverted" panel of Figure 6C: excitation is mostly along the diagonal line, while inhibition mostly flanks the diagonal area. Note that excitation is stronger at the model cell's preferred side (minus side of spatial scale), while inhibition is stronger at the model cell's null side (plus side of spatial scale). This is due to the spatial asymmetry of excitation and inhibition. The nonlinear facilitation observed in the experiment by Emerson and colleagues may derive from a specific excitatory directional interaction that is not addressed in our model or from generic nondirectional facilitations (such as network feedback or active channels on the dendrites that are masked by directional suppressions).

3.4 Layer 4 Stellate Cell Model. Although all of our parameters are physiologically plausible and the model displayed direction selectivity for a broad range of parameters, we wanted to ensure that the effect we observed was not due to the model cell's cable structure. We therefore mapped our double synaptic-veto arrangement onto an anatomically correct layer 4 stellate cell model (Mainen \& Sejnowski, 1996; see Figure 7A). All active and passive parameters of the original model cell remained unchanged. The new model's response to different types of motions is illustrated in Figure 7B. The stellate cell showed direction selectivity for normal motion and the opposite selectivity for reverse-phi motion, with DI $=1$ in both cases. We also tested our connection scheme on the layer 5 pyramidal cell model from Mainen and Sejnowski (1996). The pyramidal cell model showed the same directional preference when we mapped our synapse triples onto basal dendrites alone or basal and apical dendrites together (data not shown).

\section{Discussion}

We demonstrate here that our double synaptic-veto mechanism can account for the reversal of direction selectivity in reverse-phi motion. Our biophys- 
A

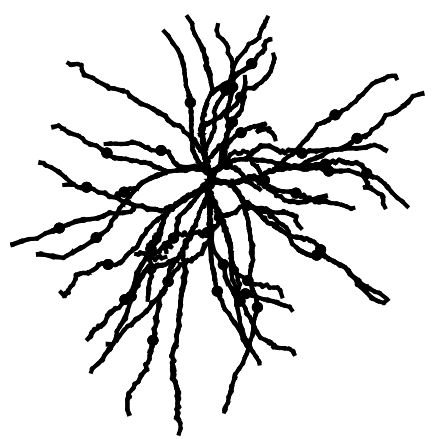

B
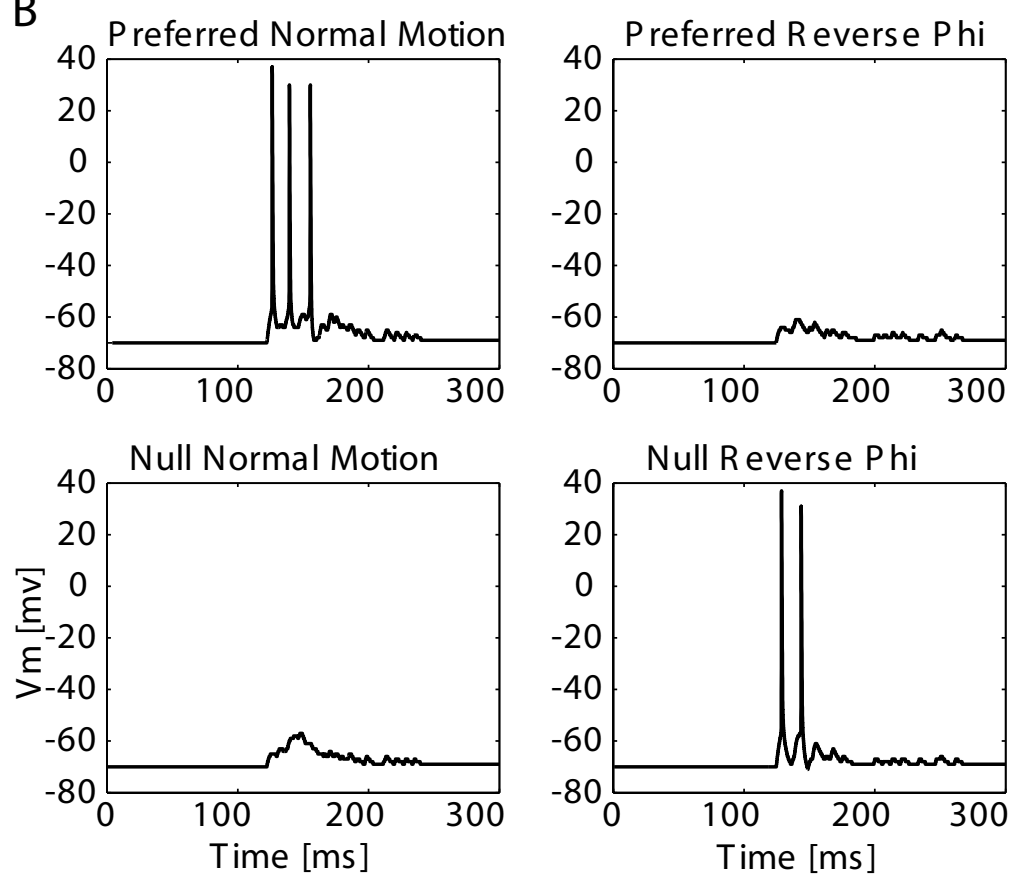

Figure 7: Mapping the double synaptic-veto mechanism onto a layer 4 stellate cell model caused it to respond differentially for normal as well as reverse-phi motion. (A) Input synapse location on a layer 4 stellate cell. (Cell model from Mainen \& Sejnowski, 1996.) (B) Stellate cell model's response to a bright bar moving at 10 degrees per second across its receptive field. The model responds best when a normal motion stimulus moves in its preferred direction and a reverse-phi motion stimulus moves in its null direction, as do many V1 cells in the macaque monkey (Livingstone et al., 2000). 
ical simulations are obviously a mere proof of concept that such a scheme might be implemented in a plausible manner by cortical cells. Given the large number of degrees of freedom of any detailed biophysical simulations and the few constraints, except for order-of-magnitude estimations on the relevant parameters, little else is possible at this time. However, the fact that different cell models with distinct dendritic morphologies and voltagedependent currents can be driven by the same synaptic arrangement to replicate the experimental data in terms of direction selectivity shows that our double synaptic-veto mechanism is not implausible from a physiological point of view.

This scheme makes several predictions that can be evaluated using extra cellular recordings. First, the nondirection-selective zone for reverse-phi motion should reside at the cell's null side instead of the preferred side. Due to our inhibitory synaptic connection scheme, the nondiscriminating zone of direction-selective cells demonstrated experimentally in retinal and cortical neurons (Livingstone, 1998; He, Jin, \& Masland, 1999) should also reverse its location for reverse-phi stimuli. Second, the response amplitude to normal motion in the preferred direction should be larger than to reversephi in the null direction (see Figures 4C and 7B). The inputs from the LGN layer to the model cell are weaker and spread into both ON and OFF channels for reverse-phi stimuli. Third, DI for reverse-phi motion should be more sensitive to parameter tuning than normal motion, especially for inhibitory input delay. Our input time course analysis (see Figures 2 and 3) suggests DI of reverse-phi motion is quite sensitive to the reversal rate: the higher the reversal rate is, the weaker are the input signals feeding into the direction-selective cell. This last property might show up in appropriate psychophysical studies as an increase in the motion detection threshold (increase of percentage of motion coherence or a decrease of $D_{\max }$ ).

In this study, we assume that direction selectivity is generated at the single cell level in a feedforward manner, without the aid of local feedback circuits. There are extensive feedback interactions among V1 cells, and these feedback currents are likely to be important for sharpening directional tuning (Douglas, Koch, Mahowald, Martin, \& Suarez, 1995; Maex \& Orban, 1996). As we stated before, our models respond in an appropriate directionselective manner to both types of motions over a broad parameter range, although DI was not always high. When DI was low, the response onset time for the preferred-direction motion was less than for the null-direction motion. A network could use this difference in response onset time to increase DI if we assume that neurons with the same direction preference have more excitatory feedback connection among themselves. In fact, this difference alone is enough to generate direction selectivity through network interaction (Maex \& Orban, 1996; Suarez et al., 1995). Excitatory feedback might also help to produce balanced response amplitude for normal-motion and reverse-phi motion. In psychophysics experiments, human subjects have the same detection threshold for normal-motion and reverse-phi motion 
(Sato, 1989). Monkey V1 cell's response amplitudes to normal motion and reverse-phi motion are also comparable (M. Livingstone, personal communication, April 2002). In our simulation, although DI = 1 for normal motion and DI $=-1$ for reverse-phi motion, our model responds with many more spikes for the normal motion due to the low-pass nature and half-wave rectification of our vision system. This difference can be decreased by network interactions if the feedforward input triggers the cortical response and sets the directional bias, while the network itself determines the amplitude of the response. Of course, the use of shunting inhibition to compute the normal and reverse-phi direction selectivity does not rule out additional biophysical mechanisms to sharpen up this selectivity (Mel, 1993; Archie \& Mel, 2000; Mel, Ruderman, \& Archie, 1998) such as facilitation in the preferred direction (Emerson et al., 1987).

We use the terms excitation and inhibition rather than facilitation or suppression in this article. Facilitation and suppression usually refer to the nonlinear part of a cell's response. We did not isolate linear responses from nonlinear responses in our analysis, although the "same minus inverted" map (see Figures $6 \mathrm{~B}$ and $6 \mathrm{C}$ ) is a plot of the nonlinear interaction and thus shows facilitation and suppression (Emerson et al., 1987; Livingstone, Pack, \& Born, 2001). The nonlinear suppression in the null direction comes from shunting inhibition. There is no significant nonlinear facilitation in our model other than NMDA synaptic inputs. This might provide positive nonlinear interaction in the real neuron. Because the excitatory input for reverse-phi motion spreads between $\mathrm{ON}$ and OFF channels, such facilitation could further increase the difference of response amplitudes. Excitatory feedback, missing in our feedforward model, might underlie the facilitation in the preferred direction and might fill in the gap of response amplitude difference between normal and reverse-phi motion.

In the traditional inhibition-based direction-selective scheme, shunting inhibition is not necessarily required if the neuron does not possess subunit structures. However, shunting inhibition is required for our double synaptic-veto mechanism to restrict the interaction to local branches. As we stated in section 3, direction selectivity for both normal and reverse-phi motion decreases to almost zero when the inhibitory channel reversal potential decreases from $-60 \mathrm{mV}$ to $-90 \mathrm{mV}$. Our double synaptic veto scheme requires branch-specific computations that cannot be achieved by nonshunting inhibition. Archie and Mel (2000) demonstrated disparity tuning and reverse-phi-like effects without shunting inhibition in a similar modeling study. The nonlinear interaction underlying disparity tuning in their model is clustering (nonlinear excitatory interaction). The basic nonlinear direction interaction in our model is between the excitation and shunting inhibition; shunting inhibition is required for the on-path veto and direction selectivity. We only used one type of GABA synapse that has a long off-ramp (80 $\mathrm{ms}$ ) in our simulation. This long off-ramp is necessary only to shut off the long-lasting NMDA currents. If we set NMDA conductance to zero and 
GABA synapse off-ramp to $2 \mathrm{~ms}$, the model is still direction selective. In real neurons, fast and slow inhibitions coexist.

The key to our double synaptic gating mechanism is that excitatory inputs are half-wave rectified and carry separate $\mathrm{ON}$ or OFF signals, while the inhibitory input carries both ON and OFF signals (in a spatially segregated manner). In our model, inhibition originates (via interneurons) from LGN ON and OFF center cells. The inhibition could, in principle, also be supplied by a cortical simple cell, with spatially offset ON and OFF regions within its receptive field. The separation of ON and OFF channels occurs at the very first mammalian visual processing stage in the retina (Rodieck, 1998). Within the mammalian visual system, it is not until V1 that these two channels combine their information. Since the detection of reverse-phi requires interaction between ON and OFF channels and the simple cell might be the first place that this interaction occurs, Livingstone et al. (2001) proposed that direction selectivity in the monkey might arise between the interactions of two simple cells that carry the full-wave signal. In the visual system of the fly, the reverse-phi effect observed in higher-order visual cells (Egelhaaf \& Borst, 1992) was used to support the argument that there is no ON-OFF channel separation. Although ON-OFF interaction is necessary to account for reverse-phi motion, our results suggest that only inhibition needs to carry the full-wave signal. The excitation to the direction-selective cell can originate from direct geniculate projection.

The synaptic triplet arrangement proposed here for our double synapticveto scheme requires rather sophisticated synapse placement during the development process. Two inhibitory ON and OFF inputs need to synapse close by their associated excitatory input or between this input and the cell body in order to be able to veto excitation effectively (Koch et al., 1982). This is in contrast to traditional shunting inhibition schemes that require only the pairing of one inhibitory process for each excitatory one. It is possible that activity-dependent, temporally asymmetric Hebbian learning rules (Markram, Lubke, Frotscher, \& Sakmann, 1997) might be used to establish such specific connection schemes as shown for normal direction selectivity by Rao and Sejnowski (2000). It does remain a major challenge to understand how direction-selective neurons with subunit structures could be established in an unsupervised manner on the basis of such learning rules.

Reverse-phi motion stimuli do not appear to be a common feature of natural spatiotemporal scenes. It therefore remains unclear why cortical cells should invert their direction selectivity for reverse-phi motion. We believe that the synaptic circuitry responsible for detecting reverse-phi motion has to be established as a by-product of developing normal direction selectivity rather than as a stand-alone training process. If the inputs to directionselective cortical neurons have a significant background firing rate, then OFF inhibition at the preferred side of ON excitation is released when a white bar moves in the preferred direction and thus helps to increase direction preference. If the inhibition comes from simple cells, it naturally 
contains spatially offset $\mathrm{ON}$ and OFF signals. The challenge is to align the $\mathrm{ON}$ and OFF regions properly with excitatory inputs. We are investigating learning rules that could account for our double synaptic-veto mechanism. The "prediction and sequence learning" mechanism proposed in Montague and Sejnowski (1994) and Montague, Dayan, Person, and Sejnowski (1995) might play an important role in the establishment of the reverse-phi motiondetection circuit.

\section{Acknowledgments}

This project originated in a joint discussion with Tomaso Poggio and Margaret Livingstone. We thank both for their help and criticism. We also thank Kevin Archie, Bartlett Mel, and Terry Sejnowski for providing us with cell models, help, and criticism. This research was supported by grants from the NSF-sponsored Engineering Research Center at Cal Tech, the National Institutes of Health, and the National Institute of Mental Health.

\section{References}

Adelson, E. H., \& Bergen, J. R. (1985). Spatiotemporal energy models for the perception of motion. J. Opt. Soc. Am. A, 2, 284-299.

Anderson, J. C., Binzegger, T., Kahana, O., Martin, K. A. C., \& Segev, I. (1999). Dendritic asymmetry cannot account for directional responses of neurons in visual cortex. Nature Neuroscience, 2, 820-824.

Anderson, J. S., Carandini, M., \& Ferster, D. (2000). Orientation tuning of input conductance, excitation, and inhibition in cat primary visual cortex. J. Neurophysiology, 84, 909-926.

Anstis, S. M. (1970). Phi movement as a subtractive process. Vision Research, 10, 1411-1430.

Anstis, S. M., \& Rogers, B. J. (1975). Illusory reversal of visual depth and movement during changes of contrast. Vision Research, 15, 957-961.

Anstis, S. M., \& Rogers, B. J. (1986). Illusory continuous motion from oscillating postive-negative patterns: Implications for motion perception. Perception, 15, 627-640.

Archie, K. A., \& Mel, B. W. (2000). A model for intradendritic computation of binocular disparity. Nature Neuroscience, 3, 54-63.

Barlow, H. B, \& Levick, R. W. (1965). The mechanism of directional selectivity in the rabbit's retina. J. Physiology, 173, 477-504.

Borg-Graham, L. J. (2001). The computation of directional selectivity in the retina occurs presynaptic to the ganglion cell. Nat Neurosci., 4, 176-183.

Borg-Graham, L. J., Monier, C., \& Frégnac, Y. (1998). Visual input evokes transient and strong shunting inhibition in visual cortical neurons. Nature, 393, 369-373.

Chubb, C., \& Sperling, G. (1989). Two motion perception mechanisms revealed through distance-driven reversal of apparent motion. Proc. Natl. Acad. Sci. USA, 86, 2985-2989. 
Conway, B. R., \& Livingstone, M. S. (2001). Space-time maps and two-bar interactions of direction-selective cells in macaque $V$-1. Manuscript submitted for publication.

Douglas, R. J., Koch, C., Mahowald, M., Martin, K. A. C., \& Suarez, H. H. (1995). Recurrent excitation in neocortical circuits. Science, 269, 981-985.

Egelhaaf, M., \& Borst, A. (1992). Are there separate ON and OFF channels in fly motion vision? Visual Neuroscience, 8, 151-164.

Emerson, R. C., Citron, M. C., Vaughn, W. J., \& Klein, S. A. (1987). Nonlinear directionally selective subunits in complex cells of cat striate cortex. J. Neurophysiology, 58, 33-65.

Gabbiani, F., Mo, C., \& Laurent, G. (2001). Invariance of angular threshold computation in a wide-field looming-sensitive neuron. J. Neuroscience, 21, 314-329.

Hatsopoulos, N., Gabbiani, F., \& Laurent, G. (1995). Elementary computation of object approach by a wide-field visual neuron. Science, 270, 10001003.

He, S., Jin, Z. F., \& Masland, R. H. (1999). The nondiscriminating zone of directionally selective retinal ganglion cells: Comparison with dendritic structure and implications for mechanism. J. Neuroscience, 19, 8049-8055.

Hines, M. L., \& Carnevale, N. T. (1997). The NEURON simulation environment. Neural Computation, 9, 1179-1209.

Horton, J. C., \& Sherk, H. (1983). Receptive field properties in the cat's lateral geniculate nucleus in the absence of ON-center retinal input. J. Neuroscience, 4, 374-380.

Ibbotson, M. R., \& Clifford, C. W. G. (2001). Interactions between ON and OFF signals in directional motion detectors feeding the NOT of the wallaby. J. Neurophysiol., 86, 997-1005.

Knapp, A. G., \& Mistler, L. A. (1983). Response properties of cells in rabbit's lateral geniculate nucleus during reversible blockade of retinal ON-center channel. J. Neurophysiology, 50, 1236-1245.

Koch, C., \& Poggio, T. (1985). The synaptic veto mechanism: Does it underlie direction and orientation selectivity in the visual cortex? In D. Rose \& V. Dobson (Eds.), Models of the visual cortex (pp. 408-419). New York: Wiley.

Koch, C., \& Poggio, T. (1987). Biophysics of computation: Neurons, synapses, and membranes. In G. M. Edelman, W. E. Gall, \& W. M. Cowan (Eds.), Synaptic function (pp. 637-697). New York: Wiley.

Koch, C., Poggio, T., \& Torre, V. (1982). Retinal ganglion cells: A functional interpretation of dendritic morphology. Philosophical Transactions of the Royal Society B, 298, 227-264.

Livingstone, M. S. (1998). Mechanisms of direction selectivity in macaque V1. Neuron, 20, 509-526.

Livingstone, M. S., Pack, C. C., \& Born, R. T. (2001). 2-D substructure of MT receptive-fields. Neuron, 30, 781-793.

Livingstone, M. S., Tsao, D. Y., \& Conway, B. R. (2000). What happens if it changes contrast when it moves? Society for Neuroscience Abstracts, 26, 162.6.

Lu, Z. L., \& Sperling, G. (1999). Second-order reversed phi. Perception and Psychophysics, 61, 1075-1088. 
Maex, R., \& Orban, G. A. (1996). Model circuit of spiking neurons generating directional selectivity in simple cells. J. Neurophysiol., 75, 1515-1545.

Mainen, Z. F., \& Sejnowski, T. J. (1996). Influence of dendritic structure on firing pattern in model neocortical neurons. Nature, 382, 363-366.

Markram, H., Lubke, J., Frotscher, M., \& Sakmann, B. (1997). Regulation of synaptic efficacy by coincidence of postsynaptic APs and EPSPs. Science, 275, 213-215.

Mather, G., \& Murdoch, L. (1999). Second-order processing of four-stroke apparent motion. Vision Research, 39, 1795-1802.

McLean, J., \& Palmer, L. A. (1989). Contribution of linear spatiotemporal receptive field structure to velocity selectivity of simple cells in area 17 of cat. Vision Res., 29, 675-679.

Mel, B. W. (1993). Synaptic integration in an excitable dendritic tree. J. Neurophysiol., 70, 1086-1101.

Mel, B. W., Ruderman, D. L., \& Archie, K. A. (1998). Translation-invariant orientation tuning in visual "complex" cells could derive from intradendritic computations. J. Neuroscience, 18, 4325-4334.

Montague, P. R, Dayan, P., Person, C., \& Sejnowski, T. J. (1995). Bee foraging in uncertain environments using predictive Hebbian learning. Nature, 377, 725-728.

Montague, P. R., \& Sejnowski, T. J. (1994). The predictive brain: Temporal coincidence and temporal order in synaptic learning mechanisms. Learn Mem, 1, $1-33$.

Poggio, T. (1982). Visual algorithms (AI Memo No. 683). Cambridge, MA: MIT Artificial Intelligence Laboratory.

Poggio, T., \& Torre, V. (1978). A new approach to synaptic interactions. In R. Heim \& G. Palm (Eds.), Theoretical approaches to complex systems (pp. 89-115). Berlin: Springer-Verlag.

Rao, R. P. N., \& Sejnowski, T. J. (2000). Predictive sequence learning in recurrent neocortical circuits. In S. A. Solla, T. K. Lee, \& K.-R. Muller (Eds.), Advances in neural information processing systems, 12 (pp. 164-170). Cambridge, MA: MIT Press.

Reichardt, W. (1961). Autocorrelation: A principle for the evaluation of sensory information by the nervous system. In W. A. Rosenblith (Ed.), Sensory communication. New York: Wiley.

Reid, R. C., Soodak, R. E., \& Shapley, R. M. (1991). Directional selectivity and spatio-temporal structure of receptive fields of simple cells in cat striate cortex. J. Neurophysiol., 66, 505-529.

Rodieck, R. W. (1998). The first steps in seeing. Sunderland, MA: Sinauer Associates.

Santen, J. P. H., \& Sperling, G. (1985). Elaborated Reichardt detectors. J. Opt. Soc. Am. A, 2, 300-320.

Sato, T. (1989). Reversed apparent motion with random dot patterns. Vision Res., 29, 1749-1758.

Schiller, P. H. (1982). Central connections of the retinal ON and OFF pathways. Nature, 297, 580-583. 
Schiller, P. H., Sandel, J. H., \& Maunsell, J. H. R. (1986). Functions of the ON and OFF channels of the visual system. Nature, 322, 824-825.

Suarez, H., Koch, C., \& Douglas, R. (1995). Modeling direction selectivity of simple cells in striate visual cortex within the framework of the canonical microcircuit. J. Neuroscience, 15, 6700-6719.

Taylor, W. R., He, S., Levick, W. R., \& Vaney, D. I. (2000). Dendritic computation of direction selectivity by retinal ganglion cells. Science, 289, 2347-2350.

Torre, V., \& Poggio, T. (1978). A synaptic mechanism possibly underlying directional selectivity to motion. Proc. Roy. Soc. Lond. B, 202, 409-416.

Wörgötter, F., \& Koch, C. (1991). A detailed model of the primary visual pathway in the cat: Comparison of afferent excitatory and intracortical inhibitory connection schemes for orientation selectivity. J. Neuroscience, 11, 1959-1979.

Received April 3, 2002; accepted October 7, 2002. 